\title{
A17
}

doi: 10.14232/ syrpharmacognosy.2021.a17

\section{Isolation and characterization of chemical constituents from Hemipholiota populnea and their bioactivities}

Morteza Yazdani

Email: morteza.yazdani@pharmacognosy.hu

Hemipholiota populnea (Pers.) Kühner ex Bon [syn. Pholiota populnea (Pers.) Kuyper \& Tjall.Beuk.] known as a member of Strophariaceae family and distributed worldwide wherever cottonwoods occur. Previously the chemistry and pharmacology of this mushroom was not investigated. Detailed phytochemical analysis of the $n$-hexane and ethyl acetate extracts of $H$. populnea led to the isolation of four lanostane derivatives (1-4) with specific ester functionality and a linear triterpene tetraol (5). Compounds 1-5 were identified as new natural products, in addition the known ergostane derivative (6) and two nucleosides (7-8) were obtained. All compounds were isolated for the first time from this mushroom. 1-3, $\mathbf{5}$ and $\mathbf{6}$ were investigated for antimicrobial activity against different bacterial strains (Gram-negative, Gram-positive, methicillin and ofloxacin resistant strains), and for cytotoxic activity against sensitive and resistant Colo 205 and Colo 320 cell lines, respectively, and against non-tumoral MRC-5 cell line. Combination with doxorubicin and efflux pump inhibitory activity of 1-3, 5 and $\mathbf{6}$ on Colo 320 cells were also assayed. No antibacterial activity could be detected, however, some of the triterpenes demonstrated remarkable cytotoxic and multidrug resistance reversal activity. Very strong synergism with doxorubicin was observed for compound $\mathbf{5}$. Our results indicate that $H$. populnea is a promising source of new terpene metabolites with significant bioactivities.

Supervisors: Attila Ványolós, Judit Hohmann

\section{Acknowledgements:}

This study was supported by National Research, Development and Innovation Office, Hungary (NKFIH; K135845). The author is grateful to Annamária Kincses and Gabriella Spengler (Department of Medical Microbiology and Immunobiology, University of Szeged) for the measurement of cytotoxicity, checkerboard combination and anti-MDR assays and assay for antibacterial activity. 